\title{
85.15 Nonlinear Inversion by Direct Search Using the Neighbourhood Algorithm
}

\author{
Malcolm Sambridge
}

Research School of Earth Sciences, Australian National University, Canberra, Australia.

\section{Background}

The Neighbourhood Algorithm (NA) was introduced by Sambridge $(1999 \mathrm{a}, \mathrm{b})$ as a direct search method for nonlinear inversion. This approach is applicable to a wide range of inversion problems, particularly those where the relationship between the observables (data) and the unknowns (a finite set of model parameters) is rather complex, e.g., fitting of seismic waveforms for Earth structure or source parameters.

The approach is divided into two stages. In the first, known as the search stage, one samples a multidimensional parameter space for combinations of parameters (models) that provide satisfactory fit to observed data. In the second, known as the appraisal stage, one tries to extract information from the complete ensemble of models collected, e.g., on resolution and trade-offs. The search algorithm is in the same class of techniques as Genetic Algorithms (GA) and Simulated Annealing (SA), in that it uses randomized decisions to drive the search and avoids the need for calculation of derivatives of the data misfit function. These techniques are often associated with global optimization problems. The NA differs from previous techniques in that it requires just two control parameters to be tuned, and the search process is driven by only the rank of models with respect to the data misfit criterion, and not the misfit itself. This allows considerable flexibility because any combination of data-fit criteria, or other information, can be used to rank models. Recently the NA has been applied to hypocenter location (Sambridge and Kennett, 2001) and seismic source characterization (MarsonPidgeon et al., 2000).

\section{The Algorithm}

The NA makes use of simple geometrical concepts to search a parameter space. The basic idea is illustrated with a simple example. Figure 1 shows results from a two-parameter problem in which the NA has been used to maximize a multipeaked fitness function in a plane. The top left panel shows the initial set of 10 points distributed quasi-randomly. At each stage, the entire parameter space is partitioned into a set of Voronoi cells (nearest neighbor regions), one about each previously sampled model. These cells are used to guide subsequent sampling in a randomized fashion. As iterations proceed, the algorithm concentrates sampling in promising regions. In this example, the NA distributes 20 new points in the best 20 Voronoi cells at each iteration. The top right panel shows the Voronoi cells after 100 points, and the bottom left after 500 points have been added. Clearly, all four prominent maxima are well sampled. In fact, the global maximum was located after 442 samples. Sambridge (1999a) has demonstrated that even though the NA is based on simple geometrical principles, it results in a highly self-adaptive search, and it remains computationally practical even in much higher dimensional spaces (e.g., 10-100).

\section{The NA-Sampler Package}

The code contributed to this volume is the NA-sampler package, which is the author's implementation of the Neighbourhood Algorithm for a multidimensional parameter space search. 


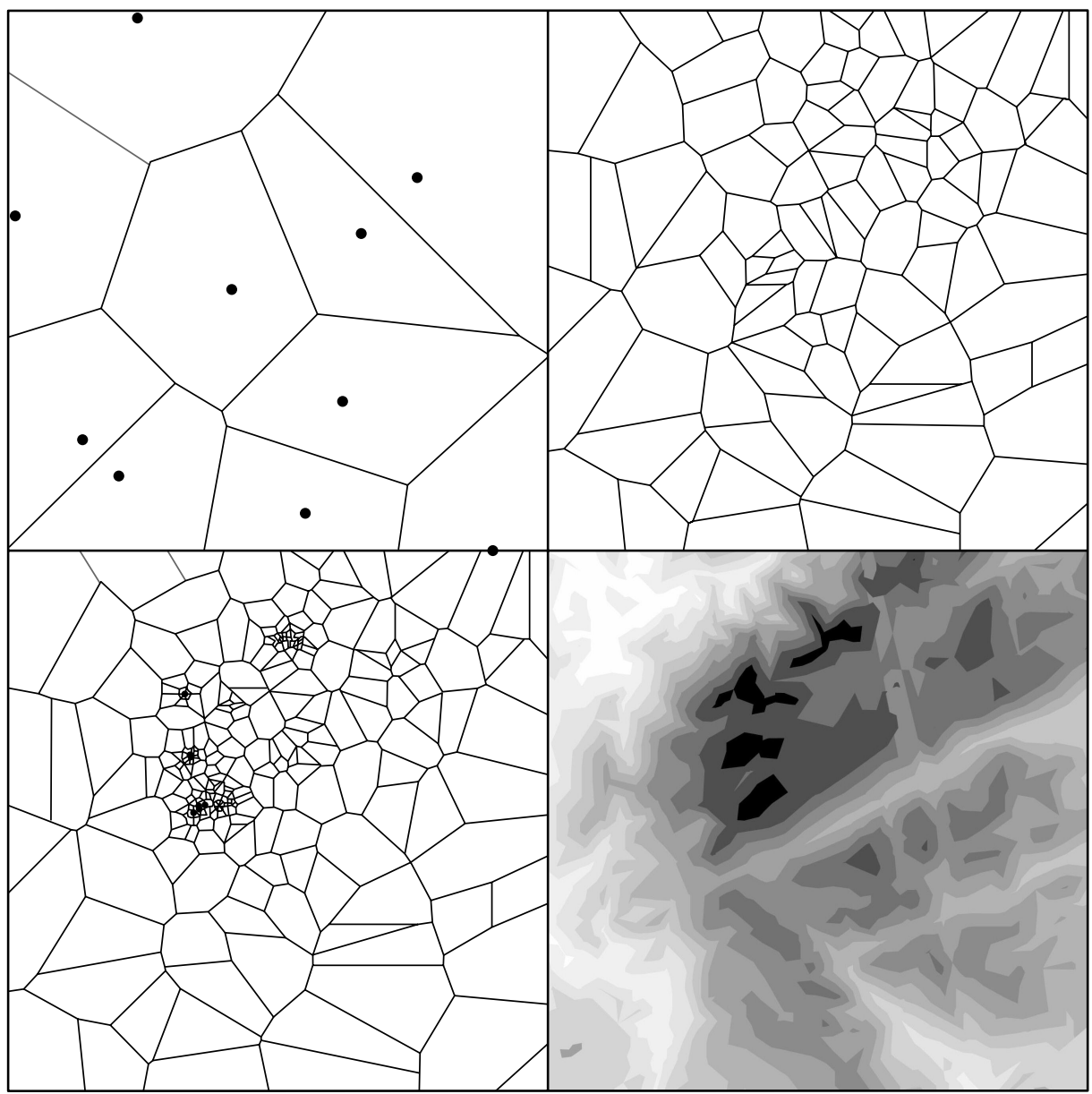

FIGURE 1 The figure shows three stages of Neighbourhood Algorithm search. The top left panel shows the initial 10 uniformly randomly distributed points and their corresponding Voronoi cells. The top right panel shows the Voronoi cells of the first 100 points generated by the NA, and the bottom left panel shows the Voronoi cells for 500 points. The bottom right panel shows the true fitness landscape; darker shades are higher fitness.

It consists of a set of user-callable subroutines and an example driver program. The latter applies the NA algorithm to the estimation of crustal seismic velocity profiles from inversion of seismic receiver functions (RFI). Several independent "utility" programs are also provided that manipulate the output files of the main program and plot some results using either X or PostScript. Some of these programs are specific to the RFI problem, and others may be more generally useful.

To apply the NA to a new problem, one needs to supply three subroutines and a main driver program that may be trivially simple, as in the case of the supplied example. These routines are compiled together with the NA code. The program is controlled by a single ASCII input file in which the two tunable parameters and other options are set. The output is in the form of a simple summary file and a direct-access binary file containing the models generated by the search algorithm together with their supplied data fit. This file can be analyzed with a plot program provided or converted to an ASCII file and assessed separately by the user. In the receiver function inversion example, one of the three user routines is used to write out all velocity models, together with the observed and predicted receiver functions from the best-fit velocity profile. Several programs are provided that display models and visually compare the data fit.

\section{Hardware and Software Requirements}

The NA-sampler package is in the subfolder for Chapter 85.15 on the attached Handbook CD. The complete source code is provided in a combination of Fortran 77 and C. Fortran coding is "standard" with no Sun Fortran or other compiler enhancements. Detailed HTML instructions are included for installing 
and running the programs. To date, the package has been compiled and tested on a Sun workstation running Solaris 2.4 with both native and GNU gcc/g77 compilers, on a Compaq Alpha running Tru64unix, and on an SGI platform running IRIX 6.5. In each case, the test examples produced exactly the same results.

\section{The NA Home Page}

The NA home page is at http://rses.anu.edu.au/ malcolm/na/ na.html. Included are instructions on obtaining the latest versions of NA-sampler as well as the author's implementation of the appraisal-stage algorithm NA-Bayes.

\section{References}

Marson-Pidgeon, K., B. L. N. Kennett, and M. Sambridge (2000). Source depth and mechanism inversion at teleseismic distances, using a neighbourhood algorithm. Bull. Seism. Soc. Am. 90, 1369-1383.

Sambridge, M. (1999a). Geophysical Inversion with a Neighbourhood Algorithm I: Searching a parameter space. Geophys. J. Int. 138, 479494.

Sambridge, M. (1999b). Geophysical Inversion with a Neighbourhood Algorithm II: Appraising the ensemble. Geophys. J. Int. 138, $727-$ 746.

Sambridge, M., and B. Kennett (2001). Seismic event location: Nonlinear inversion using a Neighbourhood Algorithm. Pure Appl. Geophys. 158, 241-257. 
